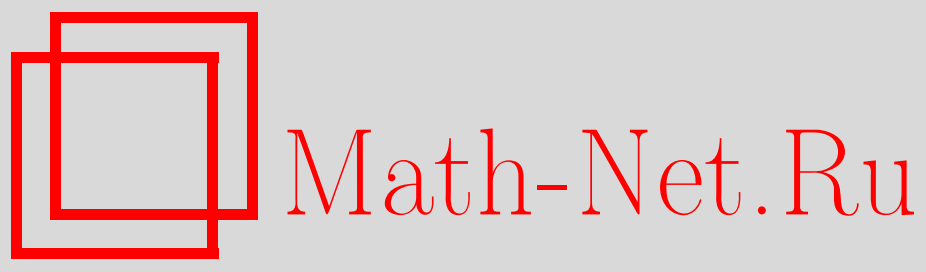

А. В. Рождественский, Точная оценка скорости сходимости в среднем биркгофовых сумм для некоторых классов периодических дифференцируемых функций, Функи. анализ и его прил., 2006, том 40, выпуск 1, 43-51

DOI: https://doi.org/10.4213/faa17

Использование Общероссийского математического портала Math-Net.Ru подразумевает, что вы прочитали и согласны с пользовательским соглашением

http://www. mathnet.ru/rus/agreement

Параметры загрузки:

IP: 54.210 .77 .194

26 апреля 2023 г., 15:01:29

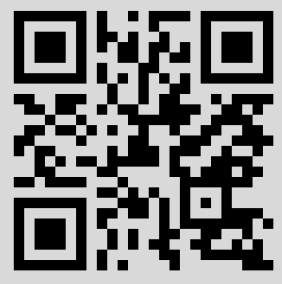


Функииональный анализ и его приложения

2006, т. 40, вып. 1, с. 43-51

УДК 517.9

\title{
Точная оценка скорости сходимости в среднем биркгофовых сумм для некоторых классов периодических дифференцируемых функций*
}

\author{
(c) 2006. А. В. РОЖДЕСТВЕНСКИй
}

\section{§1. Введение}

Пусть $\mathbb{T}^{d}=\mathbb{R}^{d} / \mathbb{Z}^{d}$ есть $d$-мерный тор, $d \in \mathbb{N}$. Тор $\mathbb{T}^{d}$ будем представлять как $d$-мерный куб $[0,1]^{d}$ с отождествленными противоположными гранями. Для заданного вектора $\alpha=\left(\alpha_{1}, \ldots, \alpha_{d}\right) \in \mathbb{R}^{d}$ через $T_{\alpha}: \mathbb{T}^{d} \rightarrow \mathbb{T}^{d}$ обозначим преобразование сдвига тора $\mathbb{T}^{d}$ на вектор $\alpha, T_{\alpha} x_{i}=x_{i}+\alpha_{i}(\bmod 1)(i=1, \ldots, d)$.

Для заданной суммируемой функции $f: \mathbb{T}^{d} \rightarrow \mathbb{R}$ и вектора $\alpha$ положим

$$
S_{n}(f)(x)=S_{n}^{\alpha}(f)(x)=\left(\sum_{s=0}^{n-1} f \circ T_{\alpha}^{s}\right)(x)=\sum_{s=0}^{n-1} f(x+s \alpha),
$$

т. е. $S_{n}(f)$ есть сумма Биркгофа порядка $n$ над преобразованием $T_{\alpha}$.

Из теоремы Кронекера-Вейля о равномерном распределении последовательности $\{s \alpha\}$ следует, что для всякой непрерывной на $\mathbb{T}^{d}$ функции $f$ биркгофовы средние $\frac{1}{n} S_{n}^{\alpha}(f)$ равномерно сходятся к «пространственному среднему» $I(f)=\int_{\mathbb{T}^{d}} f(x) d x$ тогда и только тогда, когда вектор $\alpha$ иррационален, т. е. числа $1, \alpha_{1}, \ldots, \alpha_{d}$ линейно независимы над $\mathbb{Z}$ (см. $\left.[1, \S \S 4.1,4.2]\right)$. Отсюда в силу плотности множества непрерывных функций в пространствах $L_{p}\left(\mathbb{T}^{d}\right)$ (см. [2, гл. 1 , $\S 11])$ и соотношения $\|\cdot\|_{p} \leqslant\|\cdot\|_{\infty}, 0<p<\infty$ (см. [2, гл. $\left.\left.1, \S 10\right]\right)$, немедленно следует, что средние $\frac{1}{n} S_{n}^{\alpha}(f)$ сходятся к $I(f)$ в смысле $L_{p}$-топологии для всякой функции $f \in L_{p}\left(\mathbb{T}^{d}\right), 0<p<\infty^{1)}$. Ясно, что $\frac{1}{n} S_{n}(f)-I(f)=\frac{1}{n} S_{n}(f-I(f))$, и, значит, проблема оценки скорости сходимости биркгофовых средних сводится к оценке роста суммы $S_{n}(f)$ для функций $f$ с нулевым средним. Отметим здесь, что, какой бы иррациональный вектор $\alpha$ ни взять, даже среди аналитических на $\mathbb{T}^{d}$ функций с нулевым средним найдется функция $f$, для которой величина $\left\|S_{n}^{\alpha}(f)\right\|_{p}$ не стремится к нулю при $n \rightarrow \infty$ (см. [3, гл. $\left.\left.1, \S 3\right]\right)$. С другой стороны, тривиальную оценку скорости сходимости биркгофовых средних, $\left\|S_{n}^{\alpha}(f)\right\|_{p}=o(n)\left(f \in L_{p}\left(\mathbb{T}^{d}\right), I(f)=0\right)$, нельзя уточнить для всего класса непрерывных на $\mathbb{T}^{d}$ функций. Действительно, из результатов работы [4] следует, что для всякой последовательности положительных чисел $a_{n}=o(n)$ и всякого иррационального вектора $\alpha$ найдется всюду плотное $G_{\delta}$-подмножество

*Работа выполнена при поддержке INTAS (грант 03-51-5070) и РФФИ (гранты 05-0100066, 04-01-00066).

1) Для $p \in(0,+\infty)$ и измеримой функции $f$, для которой сходится интеграл $\int_{\mathbb{T} d}|f(t)|^{p} d t$, мы полагаем $\|f\|_{p}=\left(\int_{\mathbb{T}^{d}}|f(t)|^{p} d t\right)^{1 / p}$. В случае $p \geqslant 1$ функционал $\|\cdot\|_{p}$ является нормой пространства $L_{p}\left(\mathbb{T}^{d}\right)$, а когда $p \in(0,1)-$ квазинормой, и в этом случае $\|f+g\|_{p} \leqslant$ $2^{1 / p-1}\left(\|f\|_{p}+\|g\|_{p}\right)$ для любых $f, g \in L_{p}\left(\mathbb{T}^{d}\right)$. 
непрерывных на $\mathbb{T}^{d}$ функций $f$ с нулевым средним, для которых последовательность $\frac{1}{a_{n}} S_{n}^{\alpha}(f)$ не ограничена по мере.

В данной работе для случая плохо приближаемого вектора $\alpha$ мы, во-первых, устанавливаем точную оценку $\left\|S_{n}^{\alpha}(f)\right\|_{p}=o(\sqrt{\ln n})(0<p<\infty)$ для абсолютно непрерывных периодических функций с нулевым средним и для функций из пространства бесселевых потенциалов $L_{1}^{d}\left(\mathbb{T}^{d}\right)_{0}$ (среди которых есть функции с логарифмическими особенностями), а во-вторых, для абсолютно непрерывных периодических функций с нулевым средним, у которых производная суммируема в степени $q>1$, и для функций из пространства $L_{q}^{d}\left(\mathbb{T}^{d}\right)_{0}(q>1)$ доказываем справедливость неулучшаемой оценки $\left\|S_{n}^{\alpha}(f)\right\|_{p}=O(1)(0<p<\infty)$.

\section{§2. Определения и формулировки основных результатов}

Для $x, y \in \mathbb{R}^{d}$ положим $x y=\sum_{\nu} x_{\nu} y_{\nu},|x|=|x|_{\infty}=\max _{\nu}\left|x_{\nu}\right|$, а для $z \in \mathbb{R}$ через $\|z\|$ обозначим расстояние от $z$ до ближайшего целого числа, $\|z\|=$ $\min \{\{z\}, 1-\{z\}\}$. Отметим, что $2\|z\| \leqslant|\sin \pi z| \leqslant \pi\|z\|$.

ОПРЕДЕЛЕНИЕ. Вектор $\alpha \in \mathbb{R}^{d}$ называется плохо приближсаемым, если существует положительная константа $C_{\alpha}$, такая, что

$$
|q|^{d}\|q \alpha\| \geqslant C_{\alpha} \quad\left(\forall q \in \mathbb{Z}^{d}, q \neq 0\right) .
$$

Заметим, что, согласно теореме Дирихле о совместном приближении линейных форм [5, гл. 2, §1], $\lim \inf _{|q| \rightarrow \infty}|q|^{d}\|q \alpha\| \leqslant 1$ для любого вектора $\alpha \in \mathbb{R}^{d}$.

Положим $e(y):=\exp (2 \pi i y)$ для $y \in \mathbb{R}$ и $e_{k}(x):=\exp (2 \pi i k x)$ для $x \in \mathbb{R}^{d}$, $k \in \mathbb{Z}^{d}$. Для суммируемой на $\mathbb{T}^{d}$ функции $f$ через $\hat{f}_{k}$ или $\hat{f}(k)$ будем обозначать ее коэффициенты Фурье, $\hat{f}_{k}=\hat{f}(k)=\int_{\mathbb{T}^{d}} f(t) e_{-k}(t) d t\left(k \in \mathbb{Z}^{d}\right)$.

Введем в рассмотрение следующий класс функций целого аргумента:

$$
\Theta=\left\{\theta: \mathbb{Z}^{d} \rightarrow \mathbb{C}:|\theta(k)| \equiv 1, \theta(0)=0\right\} .
$$

ОПРЕДЕЛЕНИЕ. Для $\theta \in \Theta$ через $B^{\theta}(x)=B_{d}^{\theta}(x)$ обозначим обобщенное ядро Бесселя-Макдоналъда порядка $d$ - суммируемую на $\mathbb{T}^{d}$ функцию с коэффициентами Фурье $\widehat{B^{\theta}}(k)=\theta(k)|k|^{-d}(k \neq 0)$ и $\widehat{B^{\theta}}(0)=0$.

Пусть $1 \leqslant q \leqslant \infty$. Введем в рассмотрение обобщенное пространство бесселевых потенииалов

$$
L_{q}^{d, \theta}\left(\mathbb{T}^{d}\right)=\left\{f \in L_{q}\left(\mathbb{T}^{d}\right): f(x)=I(f)+B^{\theta} * \tilde{f}(x), \tilde{f} \in L_{q}\left(\mathbb{T}^{d}\right)\right\} .
$$

Через $L_{q}^{d, \theta}\left(\mathbb{T}^{d}\right)_{0}$ будем обозначать подпространство функций $f$, для которых $I(f)=0$, пространства $L_{q}^{d, \theta}\left(\mathbb{T}^{d}\right)$. Пространство $L_{q}^{d, \theta}\left(\mathbb{T}^{d}\right)_{0}$ наделим нормой $\|f\|$ $:=\|\tilde{f}\|_{q}$. Через $B L_{q}^{d, \theta}\left(\mathbb{T}^{d}\right)_{0}$ обозначим шар радиуса единица в этом нормированном пространстве.

Еще в работе Лерха [6] было доказано, что для всякого плохо приближаемого числа $\alpha$ справедлива оценка $S_{n}^{\alpha}\left(B_{1}\right)(0)=O(\ln n)(n \rightarrow \infty)$. Из неравенства Коксмы [7, гл. 2, §5] и известной оценки отклонения последовательности $\{n \alpha\}$ для плохо приближаемого числа $\alpha[7$, гл. $2, \S 3]$ следует соотношение $\left\|S_{n}^{\alpha}\left(B_{1}\right)\right\|_{\infty}=O(\ln n)(n \rightarrow \infty)$. Из последнего, рассуждая так же, как в доказательстве теоремы 2 , заключаем, что

$$
\left\|S_{n}^{\alpha}(f)\right\|_{\infty}=o(\ln n) \quad(n \rightarrow \infty)
$$


для всякой абсолютно непрерывной функции $f$ с нулевым средним (доказательство этого соотношения содержится также в работе [4]).

Более того, в работе [4] установлена неулучшаемость последней оценки, а именно доказано, что для всякой последовательности $\left\{a_{n}\right\}$ стремящихся к нулю положительных чисел существует всюду плотное $G_{\delta}$-подмножество $W_{1}^{1}(\mathbb{T})_{0}$ функций $f$, для которых неравенство $\left\|S_{n}^{\alpha}(f)\right\|_{\infty} \geqslant a_{n} \ln n$ справедливо для бесконечно многих $n$.

Положим $R_{n}=R_{n}^{\alpha}=S_{n}^{\alpha}\left(B^{\theta}\right)$.

Нетрудно вычислить коэффициенты Фурье функции $R_{n}$. При $k \neq 0$ имеем

$$
\widehat{R}_{n}(k)=\left(R_{n} * e_{k}(x)\right) e_{-k}(x)=\theta(k) \frac{e_{k}(n \alpha)-1}{\mid k^{d}\left(e_{k}(\alpha)-1\right)} .
$$

Для заданного вектора $\alpha \in \mathbb{R}^{d}$ положим

$$
\lambda_{\alpha}(k)=\left(|k|^{d}\|\alpha k\|\right)^{-1}
$$

при $k \in \mathbb{Z}^{d} \backslash\{0\}$ и $\lambda_{\alpha}(0)=0$.

В дальнейшем нам понадобятся следующие простые оценки модулей коэффициентов Фурье функции $R_{n}(q \neq 0)$ :

$$
\begin{aligned}
& \left|\widehat{R}_{n}(q)\right| \leqslant \frac{1}{|\sin \pi \alpha q||q|^{d}} \leqslant \lambda_{\alpha}(q) \\
& \left|\widehat{R}_{n}(q)\right| \leqslant \frac{|\sin \pi n q \alpha|}{\left|q^{d}\right| \sin \pi q \alpha \mid} \leqslant \frac{\pi}{2} \frac{\|n q \alpha\|}{\left|q^{d}\right||q \alpha| \mid} \leqslant \frac{\pi}{2} \frac{n}{|q|^{d}}, \\
& \left|\widehat{R}_{n}(q)\right| \geqslant \frac{1}{(2 d)^{d / 2}} \frac{|\sin \pi n q \alpha|}{\left|q^{d}\right| \sin \pi q \alpha \mid} .
\end{aligned}
$$

В теоремах 1-4 будем предполагать, что $\alpha \in \mathbb{R}^{d}-$ плохо приближаемый вектор, а $\theta \in \Theta$.

TEOPEMA 1. Пусть $0<p<\infty$.

1. Существует положительная константа $C^{1}=C^{1}(\alpha, d, p)$, такая, что

$$
\left\|R_{n}^{\alpha}\right\|_{p} \leqslant C^{1} \sqrt{\ln (1+n)}
$$

при всех $n \in \mathbb{N}$.

2. Найдутся положительная константа $C^{2}=C^{2}(\alpha, d, p)$ и последовательность $\left\{n_{m}\right\}_{m=1}^{\infty}$ натуральньх чисел, такие, что

$$
\left\|R_{n_{m}}^{\alpha}\right\|_{p} \geqslant C^{2} \sqrt{\ln \left(1+n_{m}\right)}
$$

при всех $m \in \mathbb{N}$.

TEOPEMA 2. Пусть $0<p<\infty$.

1. Для каждой функиии $f$ из пространства $L_{1}^{d, \theta}\left(\mathbb{T}^{d}\right)_{0}$ справедливо соотношение

$$
\left\|S_{n}^{\alpha}(f)\right\|_{p}=o(\sqrt{\ln n}) \quad(n \rightarrow \infty) .
$$

2. Справедлива бормула

$$
\sup _{f \in B L_{1}^{d, \theta}\left(\mathbb{T}^{d}\right)_{0}}\left\|S_{n}^{\alpha}(f)\right\|_{p}=O(\sqrt{\ln n}) \quad(n \rightarrow \infty) ;
$$

в формуле (5) символ «O» нельзя заменить на «о». 
Последовательность натуральных чисел $\left\{n_{k}\right\}_{k=1}^{\infty}$ назовем $\alpha$-жесткой, если последовательность векторов $v_{k}=\left(n_{k} \alpha_{1}(\bmod 1), \ldots, n_{k} \alpha_{d}(\bmod 1)\right)(k=$ $1,2, \ldots$ ) стремится к нулю.

Будем говорить, что последовательность измеримых функций $g_{k}: \mathbb{T}^{d} \rightarrow \mathbb{R}-$ не ограничена по мере и писать $\mathrm{P}-\overline{\lim }_{k \rightarrow \infty} g_{k}=\infty$, если

$$
\forall A>0 \forall \varepsilon>0 \exists k: \operatorname{mes}\left\{x \in \mathbb{T}^{d}:\left|g_{k}(x)\right|>A\right\}>1-\varepsilon .
$$

Теорема 3. Пусть $1<q \leqslant \infty, f \in L_{q}^{d, \theta}\left(\mathbb{T}^{d}\right)_{0}$. Тогда для любого $p \in(0, \infty)$

$u$

$$
\left\|S_{n}^{\alpha}(f)\right\|_{p}=O(1) \quad(n \rightarrow \infty)
$$

$$
\left\|S_{n_{k}+1}^{\alpha}(f)-f\right\|_{p} \rightarrow 0 \quad(k \rightarrow \infty),
$$

для всякой $\alpha$-эсесткой последователъности $\left\{n_{k}\right\}$.

ЗАмечАниЕ. Условие на вектор $\alpha$ в теореме 3, вообще говоря, нельзя ослабить. А именно, в случае $d=1$ в работе [4] доказано, что если число $\alpha$ не является плохо приближаемым, то существует неограниченно растущая последовательность положительных чисел $\left\{a_{n}\right\}$, такая, что $\mathrm{P}-\varlimsup_{\lim } \rightarrow \infty \frac{1}{a_{n}} S_{n}^{\alpha}(f)=\infty$ для функции $f$ из некоторого всюду плотного $G_{\delta}$-подмножества пространства $W_{\infty}^{1}(\mathbb{T})_{0}$.

Последовательность натуральных чисел $\left\{n_{k}\right\}_{k=1}^{\infty}$ назовем $\alpha$-допустимой, если последовательность векторов $v_{k}=\left(n_{k} \alpha_{1}(\bmod 1), \ldots, n_{k} \alpha_{d}(\bmod 1)\right)(k=$ $1,2, \ldots$ ) имеет предел.

В теореме 4 пространство $L_{1}^{d, \theta}\left(\mathbb{T}^{d}\right)_{0}$ наделено топологией сходимости по мере.

Теорема 4. Существует $\alpha$-допустимая последовательность $\left\{n_{k}\right\}$, такая, что для каждой стремящейся $к$ нулю последовательности положительных чисел $\left\{a_{n}\right\}_{n=1}^{\infty}$ найдется всюду плотное $G_{\delta}$-подмножество функиий $f$ из $L_{1}^{d, \theta}\left(\mathbb{T}^{d}\right)_{0}$, для которьх

$$
\mathrm{P}-\varlimsup_{k \rightarrow \infty} \frac{1}{a_{n_{k}} \sqrt{\ln n_{k}}} S_{n_{k}}(f)=\infty .
$$

\section{§3. Доказательства теорем 1-4}

Для $j \in \mathbb{Z}_{+}$введем в рассмотрение «круговой слой» $\Delta_{j}=\left\{k \in \mathbb{Z}^{d}: 2^{j} \leqslant\right.$ $\left.|k|_{\infty} \leqslant 2^{j+1}-1\right\}$.

Для функции целого аргумента $\lambda: \mathbb{Z}^{d} \rightarrow \mathbb{C}$ обозначим через $\lambda^{*}(s ; \Omega)$ ее невозрастающую перестановку на множестве $\Omega \subset \mathbb{Z}^{d}$,

$$
\lambda^{*}(s ; \Omega)=\inf \{z>0: \operatorname{card}\{q \in \Omega:|\lambda(q)|>z\} \leqslant s\} .
$$

Для доказательства теоремы 1 нам понадобятся следующие три леммы, доказанные в работе [8].

Лемма А. Пусть $\alpha \in \mathbb{R}^{d}$ - плохо приблиюсаемый вектор. Тогда существует положительная константа $C$, такал, что

$$
\lambda_{\alpha}^{*}\left(s ; \Delta_{j}\right) \leqslant C / s
$$

при всех $j \in \mathbb{N}$ u $s=1, \ldots, \operatorname{card} \Delta_{j}$.

(Функция $\lambda_{\alpha}$ определена в $\S 2$.) 
Будем говорить что последовательность $\left\{q_{s}\right\}_{s=1}^{\infty} \subset \mathbb{Z}^{d}, 0<\left|q_{1}\right|<\left|q_{2}\right|<\cdots$, есть последовательность векторов наилучшего приблиюсения для $\alpha$, если $\left|q_{s}\right|^{d}\left\|q_{s} \alpha\right\| \leqslant 1$ при всех $s \in \mathbb{N}$.

Лемма В. Пусть $\alpha \in \mathbb{R}^{d}-$ плохо приблиюсаемый вектор, и пусть $\left\{q_{s}\right\}_{s=1}^{\infty}-$ последовательность векторов наилучшего приближения для $\alpha$.

Тогда для каждого $m \in \mathbb{N}$ существует иелое $n,\left|q_{m}\right|^{d} / C_{\alpha} \leqslant n \leqslant 2\left|q_{m}\right|^{d} / C_{\alpha}$, maкое, что

$$
\sum_{s=1}^{m} \frac{\sin ^{2} \pi n q_{s} \alpha}{\left|q_{s}\right|^{2 d} \sin ^{2} \pi q_{s} \alpha} \geqslant \frac{m}{4 \pi^{2}} .
$$

Лемма С. Предположим, что для функции $\lambda: \mathbb{Z}^{d} \rightarrow \mathbb{C}, \lambda(0)=0$, найдется константа $c_{1}>0$, такая, что справедливо неравенство

$$
\lambda^{*}\left(s ; \Delta_{j}\right) \leqslant c_{1} / s
$$

при всех $j \in \mathbb{N}$ us $=1, \ldots$, card $\Delta_{j}$.

Тогда для кажсдого $q \in[2, \infty)$ существуют положительные константы $C_{1}$ и $C_{2}$, такие, что для любой функиии $g \in L \log _{+}^{1 / 2} L\left(\mathbb{T}^{d}\right)$ существует функция $\Lambda g \in \bigcap_{2 \leqslant q<\infty} L^{q}\left(\mathbb{T}^{d}\right)$, коэффициентъь Фуръе которой равнъ $\lambda(k) \hat{g}_{k} u$ для которой выполняется неравенство

$$
\|\Lambda g\|_{q} \leqslant C_{1} \int_{\mathbb{T}^{d}}|g(x)| \ln ^{1 / 2}(e+|g(x)|) d x+C_{2} .
$$

(Здесь $L \log _{+}^{1 / 2} L\left(\mathbb{T}^{d}\right)$ обозначает класс суммируемых на $\mathbb{T}^{d}$ функций $g$, для которых конечен интеграл $\left.\int_{\mathbb{T} d}|g(x)| \ln ^{1 / 2}(e+|g(x)|) d x.\right)$

ДОкАЗАТЕЛЬСТво ТЕОРЕмы 1. 1. Представим функцию $R_{n}$ в виде суммы, $R_{n}(x)=R_{n}^{1}(x)+R_{n}^{2}(x)$, где $R_{n}^{1}(x)=\sum_{|q|=1}^{2^{l}-1} \widehat{R}_{n}(q) e_{q}(x), R_{n}^{2}(x)=\sum_{|q|=2^{l}}^{\infty} \widehat{R}_{n}(q) e_{q}(x)$, $l=\left[(p / d) \log _{2} n\right]+1$.

Везде ниже константы $C_{k}$ положительны и не зависят от выбора $n$. Пусть $p \in[2, \infty)$. Оценим $\left\|R_{n}^{2}\right\|_{p}$ сверху. По неравенству Хаусдорфа-Юнга

$$
\left\|R_{n}^{2}\right\|_{p}^{p^{\prime}} \leqslant \sum_{|q|=2^{l}}^{\infty}\left|\widehat{R}_{n}(q)\right|^{p^{\prime}}
$$

где $p^{\prime}=p /(p-1)$. Из неравенства (2) заключаем, что

$$
\begin{aligned}
\sum_{|q|=2^{l}}^{\infty}\left|\widehat{R}_{n}(q)\right|^{p^{\prime}} & \leqslant\left(\frac{\pi}{2}\right)^{p^{\prime}} n^{p^{\prime}} \sum_{|q|=2^{l}}^{\infty} \frac{1}{|q|^{d p^{\prime}}} \leqslant\left(\frac{\pi}{2}\right)^{p^{\prime}} n^{p^{\prime}} \sum_{s=2^{l}}^{\infty} \frac{\operatorname{card}\{q:|q|=s\}}{s^{d p^{\prime}}} \\
& \leqslant C_{1} n^{p^{\prime}} \sum_{s=2^{l}}^{\infty} \frac{1}{s^{d p^{\prime}-d+1}} \leqslant C_{2} .
\end{aligned}
$$

Теперь оценим $\left\|R_{n}^{1}\right\|_{p}$. Имеем $\operatorname{deg} R_{n}^{1} \leqslant 2^{l}-1 \leqslant n^{p / d}$. Положим $N=n^{p / d}$. Пусть $V_{N}(x)$ есть $d$-мерное ядро Валле-Пуссена степени $2 N$, т. е. $V_{N}(x)=$ $\prod_{j=1}^{d}\left(2 F_{2 N}\left(x_{j}\right)-F_{N}\left(x_{j}\right)\right), F_{N}\left(x_{j}\right)=1+2 \sum_{k=1}^{N}(1-k / N) \cos 2 \pi k x_{j}$. Из определения ядра $V_{N}(x)$ немедленно следует, что $\widehat{V}_{N}(q)=1$, когда $|q| \leqslant N$, а из известных свойств ядра Фейера $F_{N}$ заключаем, что $1 \leqslant\left\|V_{N}\right\|_{1} \leqslant 3^{d},\left\|V_{N}\right\|_{\infty} \leqslant 2^{d} N^{d}$. 
Из последних двух неравенств и определения числа $N$ следует, что

$$
\int_{\mathbb{T}^{d}}\left|V_{N}(x)\right| \ln ^{1 / 2}\left(1+\left|V_{N}(x)\right|\right) d x \leqslant 3^{d} \ln ^{1 / 2}\left(1+2^{d} N^{d}\right) \leqslant C_{3} \sqrt{\ln (1+N)} .
$$

Из неравенства (1) и леммы А заключаем, что коэффициенты полинома $R_{n}^{1}$ удовлетворяют условиям леммы С. В силу этой леммы и последнего неравенства

$$
\left\|R_{n}^{1} * V_{N}\right\|_{p} \leqslant C_{4} \int_{\mathbb{T}^{d}}\left|V_{N}(x)\right| \ln ^{1 / 2}\left(1+\left|V_{N}(x)\right|\right) d x+C_{5} \leqslant C_{6} \sqrt{\ln (1+n)} .
$$

Отсюда, учитывая равенство $R_{n}^{1}(x)=R_{n}^{1} * V_{N}(x)$, заключаем, что $\left\|R_{n}^{1}\right\|_{p} \leqslant$ $C_{6} \sqrt{\ln (1+n)}$.

Итак, для $p \in[2, \infty)$ мы доказали неравенство

$$
\left\|R_{n}\right\|_{p} \leqslant\left\|R_{n}^{1}\right\|_{p}+\left\|R_{n}^{2}\right\|_{p} \leqslant C_{7} \sqrt{\ln (1+n)},
$$

а для $p \in(0,2)$ воспользуемся тривиальной оценкой $\left\|R_{n}\right\|_{p} \leqslant\left\|R_{n}\right\|_{2}$.

2. Докажем теперь п. 2 теоремы 1.

Из теоремы Дирихле о совместном приближении линейных форм следует, что для всякого плохо приближаемого $d$-мерного вектора существуют последовательность векторов наилучшего приближения $\left\{q_{k}\right\}_{k=1}^{\infty}$ и константа $E>0$, такие, что

$$
\left|q_{k}\right| \leqslant E^{k} \quad(k=1,2, \ldots) .
$$

Из неравенства (3) и леммы В вытекает, что существует последовательность $\left\{n_{m}\right\}_{m=1}^{\infty}$, для которой

$\left\|R_{n_{m}}\right\|_{2}^{2} \geqslant \sum_{s=1}^{m}\left|\widehat{R}_{n_{m}}\left(q_{s}\right)\right|^{2} \geqslant \frac{1}{(2 d)^{d}} \sum_{s=1}^{m} \frac{\sin ^{2} \pi n_{m} q_{s} \alpha}{\left|q_{s}\right|^{2 d} \sin ^{2} \pi q_{s} \alpha} \geqslant \frac{m}{4(2 d)^{d} \pi^{2}} \quad(m=1,2, \ldots)$ и $\left|q_{m}\right|^{d} \leqslant C_{\alpha} n_{m} \leqslant 2\left|q_{m}\right|^{d}$. Отсюда, имея в виду неравенство $(9)$, заключаем, что

$$
\left\|R_{n_{m}}\right\|_{2} \geqslant C_{9} \sqrt{\ln \left(1+n_{m}\right)} \quad(m=1,2, \ldots) .
$$

Если $2<p<\infty$, то $\left\|R_{n_{m}}\right\|_{p} \geqslant\left\|R_{n_{m}}\right\|_{2} \geqslant C_{9} \sqrt{\ln \left(1+n_{m}\right)}(m=1,2, \ldots)$.

Пусть теперь $0<p<2$. Определим $\theta \in(0,1)$ из уравнения $1 / 2=\theta / p+$ $(1-\theta) / 4$. В силу выпуклости функции $\eta(x):=\ln \left\|R_{n_{m}}\right\|_{1 / x}, x \in(0,+\infty)$ (см. [2, гл. $1, \S 10])$, имеет место неравенство $\left\|R_{n_{m}}\right\|_{2} \leqslant\left\|R_{n_{m}}\right\|\left\|_{p}^{\theta}\right\| R_{n_{m}} \|_{4}^{1-\theta}$. Поэтому $\left\|R_{n_{m}}\right\|_{p} \geqslant\left\|R_{n_{m}}\right\|_{2}^{1 / \theta}\left\|R_{n_{m}}\right\|_{4}^{1-1 / \theta}$. Отсюда, используя неравенства (8) и (10), получаем, что

$$
\left\|R_{n_{m}}\right\|_{p} \geqslant C_{10} \sqrt{\ln \left(1+n_{m}\right)} \quad(m=1,2, \ldots) .
$$

ДокаЗАТЕльство теоремы 2. 1. Пусть $f \in L_{1}^{d, \theta}\left(\mathbb{T}^{d}\right)_{0}$. Из определения пространства $L_{1}^{d, \theta}\left(\mathbb{T}^{d}\right)$ следует, что найдется функция $g \in L_{1}\left(\mathbb{T}^{d}\right), I(g)=0$, такая, что $f(x)=B_{d}^{\theta} * g(x)$ (для п. в. $x$ ) и, значит, $S_{n}(f)(x)=R_{n} * g(x)$ (для п. в. $x)$.

Положим $K_{n}(x)=\ln ^{-1 / 2}(1+n) R_{n}(x)(n=1,2, \ldots)$.

Пусть сначала $p \in[1, \infty)$. В силу п. 1 теоремы 1 найдется положительная константа $C_{1}$, такая, что для любых $h \in L_{1}\left(\mathbb{T}^{d}\right)$ и $n \in \mathbb{N}$

$$
\left\|K_{n} * h\right\|_{p} \leqslant\left\|K_{n}\right\|_{p}\|h\|_{1} \leqslant C_{1}\|h\|_{1} .
$$


Зафиксируем $\varepsilon>0$. Пусть $t_{\varepsilon}-$ тригонометрический полином с нулевым свободным коэффициентом, такой, что $\left\|g-t_{\varepsilon}\right\|_{1}<\varepsilon /\left(2 C_{1}\right)$.

Из формулы (1) и определения функции $\lambda_{\alpha}$ следует, что величины $\left|\widehat{R}_{n}(q)\right|$ равномерно ограничены по $n$ и $q$. Поэтому

$$
\left\|K_{n} * t_{\varepsilon}\right\|_{p} \leqslant \ln ^{-1 / 2}(1+n) \sum_{q}\left|\widehat{R}_{n}(q)\right|\left|\hat{t}_{\varepsilon}(q)\right| \leqslant C_{2} \ln ^{-1 / 2}(1+n),
$$

где $C_{2}>0$ не зависит от выбора $n$. Следовательно, существует $N \in \mathbb{N}$, такое, что при всех $n \geqslant N$ справедливо неравенство $\left\|K_{n} * t_{\varepsilon}\right\|_{p} \leqslant \varepsilon / 2$. Поэтому из (11) заключаем, что для всякого $\varepsilon>0$ найдется $N \in \mathbb{N}$, такое, что при всех $n \geqslant N$

$$
\left\|K_{n} * g\right\|_{p} \leqslant\left\|K_{n} *\left(g-t_{\varepsilon}\right)\right\|_{p}+\left\|K_{n} * t_{\varepsilon}\right\|_{p} \leqslant C_{1}\left\|g-t_{\varepsilon}\right\|_{1}+\varepsilon / 2 \leqslant \varepsilon .
$$

Следовательно,

$$
\left\|\ln ^{-1 / 2}(1+n) S_{n}(f)\right\|_{p}=o(1) \quad(n \rightarrow \infty) .
$$

Теперь, чтобы доказать соотношение (4) для $p \in(0,1)$, воспользуемся свойством монотонного неубывания (по параметру $p$ ) величины $\left\|S_{n}(f)\right\|_{p}$.

2. Формула (5) непосредственно следует из соотношения (11). Пусть последовательность $\left\{n_{m}\right\}$ и константа $C^{2}$ - те, существование которых доказано в п. 2 теоремы 1. Введем в рассмотрение функцию

$$
\chi_{\varepsilon}= \begin{cases}(2 \varepsilon)^{-d}, & \text { если }|x|<\varepsilon, \\ 0 & \text { в противном случае. }\end{cases}
$$

Ясно, что $\left\|\chi_{\varepsilon}\right\|_{1}=1$. Если $1 \leqslant p<\infty$ и $g \in L_{p}\left(\mathbb{T}^{d}\right)$, то $\left\|g-g * \chi_{\varepsilon}\right\|_{p} \rightarrow 0$ при $\varepsilon \rightarrow 0$ (см. [9, гл. $1, \S 1])$. Следовательно, для каждого $m$ и любого $p \in[1, \infty)$ найдется константа $\varepsilon_{m, p}>0$, такая, что $\left\|R_{n_{m}}-R_{n_{m}} * \chi_{\varepsilon_{m, p}}\right\|_{p}<\frac{1}{2}\left\|R_{n_{m}}\right\|_{p}$. Отсюда следует, что

$$
\left\|R_{n_{m}} * \chi_{\varepsilon_{m, p}}\right\|_{p} \geqslant\left\|R_{n_{m}}\right\|_{p}-\left\|R_{n_{m}}-R_{n_{m}} * \chi_{\varepsilon_{m, p}}\right\|_{p}>\frac{1}{2}\left\|R_{n_{m}}\right\|_{p}
$$

при всех $p \in[1, \infty)$ и $m \in \mathbb{N}$. В случае $p \in(0,1)$ воспользуемся неравенствами $\left\|R_{n_{m}}\right\|_{p} \leqslant 2^{1 / p-1}\left(\left\|R_{n_{m}} * \chi_{\varepsilon}\right\|_{p}+\left\|R_{n_{m}}-R_{n_{m}} * \chi_{\varepsilon}\right\|_{p}\right),\left\|R_{n_{m}}-R_{n_{m}} * \chi_{\varepsilon}\right\|_{p} \leqslant \| R_{n_{m}}-$ $R_{n_{m}} * \chi_{\varepsilon} \|_{1}$ и выберем $\varepsilon_{m, p}>0$ так, чтобы $\left\|R_{n_{m}}-R_{n_{m}} * \chi_{\varepsilon_{m, p}}\right\|_{1}<2^{-1 / p}\left\|R_{n_{m}}\right\|_{p}$.

Теперь, полагая $f_{m, p}:=B^{\theta} * \chi_{\varepsilon_{m, p}}$, из п. 2 теоремы 1 заключаем, что

$$
\begin{aligned}
\frac{1}{\sqrt{\ln n_{m}}}\left\|S_{n_{m}}^{\alpha}\left(f_{m, p}\right)\right\|_{p} & =\frac{1}{\sqrt{\ln n_{m}}}\left\|R_{n_{m}}^{\alpha} * \chi_{\varepsilon_{m, p}}\right\|_{p} \\
& \geqslant \frac{1}{2^{\max (1,1 / p)}} \frac{1}{\sqrt{\ln n_{m}}}\left\|R_{n_{m}}^{\alpha}\right\|_{p} \geqslant \frac{C^{2}}{2^{\max (1,1 / p)}}
\end{aligned}
$$

(и при этом $\left.f_{m, p} \in B L_{1}^{d, \theta}\left(\mathbb{T}^{d}\right)_{0}\right)$, что доказывает п. 2 теоремы 2.

ДокАЗАтеЛЬСтво теоремы 3. В работе [8] доказано, что для всякой функции $f$ из пространства $L_{q}^{d, \theta}\left(\mathbb{T}^{d}\right)_{0}(q>1)$ найдется функция $w \in \bigcap_{1 \leqslant p<\infty} L_{p}\left(\mathbb{T}^{d}\right)$, такая, что $f=w \circ T_{\alpha}-w$.

Отсюда заключаем, что $S_{n}^{\alpha}(f)=w \circ T_{\alpha}^{n}-w$ и

$$
\left\|S_{n}^{\alpha}(f)\right\|_{p}=\left\|w \circ T_{\alpha}^{n}-w\right\|_{p} \leqslant \begin{cases}2\|w\|_{p}, & \text { если } p \geqslant 1, \\ 2^{1 / p}\|w\|_{p}, & \text { если } 0<p<1,\end{cases}
$$


при всех $n \in \mathbb{N}$. Далее,

$$
\begin{aligned}
\left\|S_{n_{k}+1}^{\alpha}(f)-f\right\|_{p} & =\left\|w \circ T_{\alpha}^{n_{k}+1}-w-f\right\|_{p}=\left\|w \circ T_{\alpha}^{n_{k}+1}-w \circ T_{\alpha}\right\|_{p} \\
& =\left\|w \circ T_{\alpha}^{n_{k}}-w\right\|_{p} \rightarrow 0 \quad(k \rightarrow \infty),
\end{aligned}
$$

где последнее свойство является следствием того факта, что непрерывные функции всюду плотны в пространстве $L_{p}\left(\mathbb{T}^{d}\right), 0<p<\infty$.

ДоКАЗАТЕЛЬСТво ТЕОРЕмЫ 4. Пусть последовательность $\left\{n_{m}\right\}$ - та, существование которой доказано в п. 2 теоремы 1 . Выберем из нее $\alpha$-допустимую подпоследовательность $\left\{n_{m_{k}}\right\}$, которую для простоты записи обозначим через $\left\{n_{k}\right\}$. Положим $K_{k}(f)=S_{n_{k}}(f) /\left(a_{n_{k}} \sqrt{\ln n_{k}}\right), H=\left(L_{1}^{d, \theta}\left(\mathbb{T}^{d}\right)_{0}, \rho_{0}\right)$, где

$$
\rho_{0}(f, g)=\int_{\mathbb{T}^{d}} \frac{|f(x)-g(x)|}{1+|f(x)-g(x)|} d x
$$

- метрика, индуцирующая топологию сходимости по мере.

Положим $U=\left\{f \in H: \mathrm{P}-\overline{\lim }_{k \rightarrow \infty} K_{k}(f)=\infty\right\}$.

Докажем, что $U \neq \varnothing$. Пусть $\left\{T_{k}\right\}_{k=1}^{\infty} \subset L_{2}\left(\mathbb{T}^{d}\right)$ - заданная последовательность функций. В работах [10] и [11] доказано, что если

$$
\forall A>0 \forall B>0 \quad \exists k \in \mathbb{N}:\left\|T_{k}\right\|_{1}>A+B \ln \left(1+\left\|T_{k}\right\|_{2}\right),
$$

то для некоторой функции $g_{0} \in L_{1}\left(\mathbb{T}^{d}\right)$ последовательность $\left\{T_{k} * g_{0}\right\}_{k=1}^{\infty}$ не ограничена по мере.

Положим $T_{k}(x)=K_{k}\left(B^{\theta}\right)(x)$. Заметим, что если $f_{0}=B^{\theta} * g_{0}$, то $K_{k}\left(f_{0}\right)=$ $K_{k}\left(B^{\theta} * g_{0}\right)=K_{k}\left(B^{\theta}\right) * g_{0}=T_{k} * g_{0}$ в силу перестановочности операций свертки и сдвига.

Из теоремы 1 следует, что таким образом выбранная последовательность функций $\left\{T_{k}\right\}$ удовлетворяет условию (12). Следовательно, найдется функция $g_{0} \in L_{1}\left(\mathbb{T}^{d}\right)$, такая, что последовательность $\left\{T_{k} * g_{0}\right\}_{k=1}^{\infty}$ не ограничена по мере.

Положим $f_{0}=B^{\theta} * g_{0}$. Тогда $f_{0} \in H, K_{k}\left(f_{0}\right)=T_{k} * g_{0}$ и, значит, $\mathrm{P}-\overline{\lim }_{k \rightarrow \infty} K_{k}\left(f_{0}\right)$ $=\infty$, что и требовалось.

Докажем теперь, что $U$ - всюду плотное подмножество в $H$. Без ограничения общности можно считать, что $\lim _{n \rightarrow \infty} a_{n} \sqrt{\ln n}=\infty$.

Пусть $f_{0} \in U$, а $p$ - произвольный тригонометрический полином с нулевым свободным членом. Из того, что $\alpha$ - плохо приближаемый вектор, в частности, следует, что числа $1, \alpha_{1}, \ldots, \alpha_{d}$ линейно независимы над $\mathbb{Z}$ и, значит, найдется полином $\tau$, такой, что $p(x)=\tau\left(T_{\alpha} x\right)-\tau(x)$.

Поэтому $K_{k}(p)(x)=\left(\tau\left(T_{\alpha}^{n_{k}} x\right)-\tau(x)\right) /\left(a_{n_{k}} \sqrt{\ln n_{k}}\right)$, а значит, $\left\|K_{k}(p)\right\|_{\infty} \rightarrow 0$ при $n \rightarrow \infty$.

Отсюда и из предположения о $f_{0}$ немедленно следует, что $\mathrm{P}-\overline{\lim }_{k \rightarrow \infty} K_{k}\left(f_{0}+\right.$ p) $=\infty$.

В силу произвольности выбора полинома $p$ последнее равенство означает, что множество $U$ всюду плотно в $H$.

Наконец, докажем, что $U-$ множество типа $G_{\delta}$.

Для измеримой функции $h$ и положительного числа $t$ положим $E(h, t)=$ $\operatorname{mes}\{x:|h(x)|>t\}$. Нетрудно доказать, что $E(h, t)$ - непрерывная справа функция от $t$ и что для любых измеримых $h_{1}, h_{2}$ и положительных $t_{1}$ и $t_{2}$ справедливо неравенство

$$
E\left(h_{1} \pm h_{2}, t_{1}+t_{2}\right) \leqslant E\left(h_{1}, t_{1}\right)+E\left(h_{2}, t_{2}\right) .
$$


Отметим также, что $E(h, t) \leqslant(1+1 / t) \rho_{0}(h, 0)$.

Представим $U$ в виде $U=\bigcap_{N, m=1}^{\infty} U_{N, m}$, где

$$
U_{N, m}=\left\{f \in H: \exists k E\left(K_{k}(f), N\right)>1-1 / m\right\},
$$

и докажем, что каждое из множеств $U_{N, m}$ открыто в $H$.

Зафиксируем $N, m, f \in U_{N, m}$ и определим $k$ из условия $E\left(K_{k}(f), N\right)>$ $1-1 / m$. Положим $\sigma=E\left(K_{k}(f), N\right)$. В силу непрерывности справа функции $E\left(K_{k}(f), \cdot\right)$ найдется такое $\delta>0$, что $E\left(K_{k}(f), N+\delta\right)>\frac{1}{2}(\sigma+1-1 / m)$.

Тогда в силу (13) имеем

$$
E\left(K_{k}(f), N+\delta\right) \leqslant E\left(K_{k}(f+g), N\right)+E\left(K_{k}(g), \delta\right) .
$$

При этом

$$
\begin{aligned}
E\left(K_{k}(g), \delta\right) & =E\left(\sum_{s=0}^{n-1} g \circ T_{\alpha}^{s}, \delta a_{n} \sqrt{\ln n}\right) \leqslant \sum_{s=0}^{n-1} E\left(g \circ T_{\alpha}^{s}, \frac{\delta a_{n} \sqrt{\ln n}}{n}\right) \\
& =n E\left(g, \frac{a_{n} \sqrt{\ln n} \delta}{n}\right) \leqslant n\left(1+\frac{n}{\delta a_{n} \sqrt{\ln n}}\right) \rho_{0}(g, 0) .
\end{aligned}
$$

Значит, при достаточно малом $\varepsilon>0$ и $g \in H$, таком, что $\rho_{0}(g, 0)<\varepsilon$, имеем $E\left(K_{k}(g), \delta\right)<\frac{1}{2}(\sigma-1+1 / m)$. Следовательно, для такого $g$

$$
\begin{aligned}
E\left(K_{k}(f+g), N\right) & \geqslant E\left(K_{k}(f), N+\delta\right)-E\left(K_{k}(g), \delta\right) \\
& >\frac{1}{2}\left(\sigma+1-\frac{1}{m}\right)-\frac{1}{2}\left(\sigma-1+\frac{1}{m}\right)=1-\frac{1}{m},
\end{aligned}
$$

т. е. $f+g \in U_{N, m}$.

Автор признателен Б. С. Кашину и Н. Г. Мощевитину за полезные обсуждения.

\section{ЛитерАТУРА}

1. Каток А. Б., Хасселблат Б. Введение в современную теорию динамических систем. Факториал, М., 1999.

2. Зигмунд А. Тригонометрические ряды. Т. 1, 2, Мир, М., 1965.

3. Коробов Н. M. Теоретикочисловые методы в приближенном анализе. Физматлит, M., 1963.

4. Liardet P., Volny D. Sums of continuous and differentiable functions in dynamical systems. Israel J. Math., 98, 29-60 (1997).

5. Шмидт В. М. Диофантовы приближения. Мир, М., 1983.

6. Lerch M. Question 1547. L'Intermediaire Math., 11, 144-145 (1904).

7. Кейперс Л., Нидеррейтер Г. Равномерное распределение последовательностей. Наука, М., 1985.

8. Рождественский $A$. B. Об аддитивном когомологическом уравнении и замене времени в линейном потоке на торе с диофантовым вектором частот. Матем. сб., 195, №5, 115-156 (2004).

9. Стейн И., Вейс Г. Введение в гармонический анализ на евклидовых пространствах. Мир, М., 1974.

10. Пичугов C. A. Последовательности ограниченных по мере операторов. Матем. сб., 185, № 1, 43-72 (1994).

11. Пичугов C. А. Оценки норм операторов, непрерывных по мере. Матем. заметки, 50, № $1,146-148$ (1991)

Московский государственный университет, механико-математический факультет 